\title{
Dynamic Water Promotes Lithium Ion Transport in Superconcentrated and Eutectic Aqueous Electrolytes
}

Jungyu Kim ${ }^{1,2}$, Bonhyeop Koo ${ }^{3}$, Joonhyung Lim ${ }^{1}$, Jonggu Jeon ${ }^{1}$, Chaiho Lim ${ }^{1,2}$, Hochun Lee ${ }^{3, *}$, Kyungwon Kwak ${ }^{1,2, *}$, and Minhaeng Cho ${ }^{1,2, *}$

${ }^{1}$ Center for Molecular Spectroscopy and Dynamics, Institute for Basic Science (IBS), Seoul 02841, Korea

${ }^{2}$ Department of Chemistry, Korea University, Seoul 02841, Korea

${ }^{3}$ Department of Energy Science and Engineering, DGIST, Daegu 42988, Korea

Supplemental Note 1. Experimental methods

Supplemental Note 2. Peak assignment of two water species in FTIR spectroscopy

Supplemental Note 3. MD simulation and analysis methods

Figure S1. Spectrum of MIR pulse laser and OD stretching band in $28 \mathrm{~m}$ eutectic electrolyte

Figure S2. FTIR analysis of OD stretching band in LiTFSI \& eutectic electrolytes in $5 \mathrm{v} \%$ HOD solutions

Figure S3. OH-bond rotational dynamics from MD simulations

Figure S4. Frequency-dependent transition dipole of various concentrated LiTFSI \& eutectic electrolytes in $5 \mathrm{v} \%$ HOD water solution

Figure S5. IR-PP spectra of OD stretching band in various concentrated LiTFSI and eutectic electrolytes at $0.2 \mathrm{ps}$

Figure S6. Fitting analyses of isotropic IR-PP signals in LiTFSI electrolytes

Figure S7. Fitting analyses of isotropic IR-PP signals in eutectic electrolytes

Figure S8. Anisotropic signal decay pattern of OD stretching mode in eutectic \& LiTFSI electrolytes

Figure S9. DRS spectra and deconvoluted peaks of water and LiTFSI electrolytes

Figure S10. DRS spectra and deconvoluted peaks of water and eutectic electrolytes

Figure S11. The concentration of slow water and bulk-like water in LiTFSI \& eutectic electrolytes

Figure S12. The viscosity of LiTFSI and eutectic electrolytes

Table S1. Intramolecular force field parameters of the BETI anion

Table S2. Partial charges of all ionic species in the aqueous solutions of eutectic mixture Li(TFSI $)_{0.7}(\mathrm{BETI})_{0.3}$ 
Table S3. Distribution of atomic composition in the first solvation shells of atoms, OH groups, and water molecules in the $21 \mathrm{~m}$ electrolyte

Table S4. Biexponential fitting result of the rotational TCF

Table S5. Fitted parameters of DRS for LiTFSI electrolytes

Table S6. Fitted parameters of DRS for eutectic electrolytes

Reference 
Supplemental Note 1. Experimental methods

FTIR and mid-IR pump-probe. For FTIR absorption and IR-PP spectroscopy, a liquid sample was sandwiched between two flat $\mathrm{CaF}_{2}$ windows (diameter $25 \mathrm{~mm}$, thickness $3 \mathrm{~mm}$ ). A Teflon spacer was used to adjust the path length of the sample cell. The IR absorption spectra were measured with Frontier MIR Spectrometer (PerkinElmer). For each FTIR measurement, 16 scans were performed. The frequency resolution was set to $4 \mathrm{~cm}^{-1}$. Experimental details of our IR PP setup was already presented before. Ti:Sapphire oscillator (Maitai, Spectra Physics) and a regenerative amplifier (SolsticeAce, Spectra Physics) produce $800 \mathrm{~nm}$ laser pulses with a repetition frequency of $1 \mathrm{kHz}$. BBO crystal-based optical parametric amplifier (OPA) system generates two near-IR pulses with wavelengths of approximately 1.3 and $2.0 \mu \mathrm{m}$. Mid-IR (MIR) pulses with a center wavenumber of $2500 \mathrm{~cm}^{-1}$ are generated via a difference-frequencygeneration process induced by an $\mathrm{AgGaS}_{2}$ crystal. The generated MIR pulse has an approximately $35 \mathrm{fs}$ pulse duration time and about $29 \mathrm{uJ}$ energy. The MIR pulse spectrum is shown in Fig.S1. Note that the Ge plate was used to remove the two near IR pulses (which are generated by OPA, signal and idler pulses). Furthermore, the thicknesses of Ge plates and $\mathrm{CaF}_{2}$ windows are controlled to compensate for chirp between pump and probe pulses. To carry out polarization-selective IR-PP measurements, we used a ZnSe beam splitter (9:1) to produce both pump and probe pulses. The repetition rate of the pump pulse was adjusted to be $500 \mathrm{~Hz}$ using an electronically synchronized optical chopper. The two MIR pulses are illuminated on samples through different beam paths and the time delay between pulses was controlled via retroreflectors mounted on motorized delay stages. To obtain the rotational dynamics of vibrational transition dipole, polarization-selective measurements are necessary. With wire grid polarizers, the polarization angle between probe and pump pulse is set to be $45^{\circ}$, making the probe pulse consist of identical contributions of parallel $\left(0^{\circ}\right)$ and perpendicular $\left(90^{\circ}\right)$ components with respect to pump polarization. After the interactions of pump and probe pulses with the sample, the probe pulse was divided into parallel and perpendicular polarization components with respect to the pump polarization using $\mathrm{ZnSe}$ beam splitter (5:5) and wire grid polarizer. The pump-induced changes in vibrational states in sample molecules can be monitored by the probe pulse, which is measured by a monochromator (Horiba, iHR320) and $128(64 * 2)$-element array MCT detector. The parallel polarization component of the probe beam was detected by the bottom array of the dual array MCT detector. In contrast, the perpendicular polarization component of the probe beam was detected by the upper array. We measured both the 0-1 transition (ground-state bleach and stimulated emission contributions to the IR PP) and the 1-2 transition (excited-state absorption contribution to the IR-PP) of the OD stretching vibration. Due to the overlap of $\mathrm{CO}_{2}$ absorption band and 1-2 transition of OD stretching band, the entire IR-PP setup was purged with $\mathrm{N}_{2}$ gas to remove the mid-IR absorption by gaseous $\mathrm{CO}_{2}$ in the air. With polarization selective IR-PP signals (i.e. parallel $\left(\Delta \alpha_{\|}\right)$and perpendicular $\left.\operatorname{signal}\left(\Delta \alpha_{\perp}\right)\right)$, one can obtain the vibrational population relaxation and orientational relaxation of HOD molecules, ${ }^{1}$ at the same time. The equations of the isotropic (vibrational relaxation) and anisotropic (rotational relaxation) signals are given by

$P(t)=\frac{\Delta \alpha_{\|}+2 \Delta \alpha_{\perp}}{3}$ 
$r(t)=\frac{\Delta \alpha_{\|}-\Delta \alpha_{\perp}}{\Delta \alpha_{\|}+2 \Delta \alpha_{\perp}}=0.4 C_{2}(t)$

where $P(t)$ is the vibrational population relaxation, $r(t)$ is the anisotropy and $C_{2}(t)$ is the second Legendre polynomial orientational correlation function.

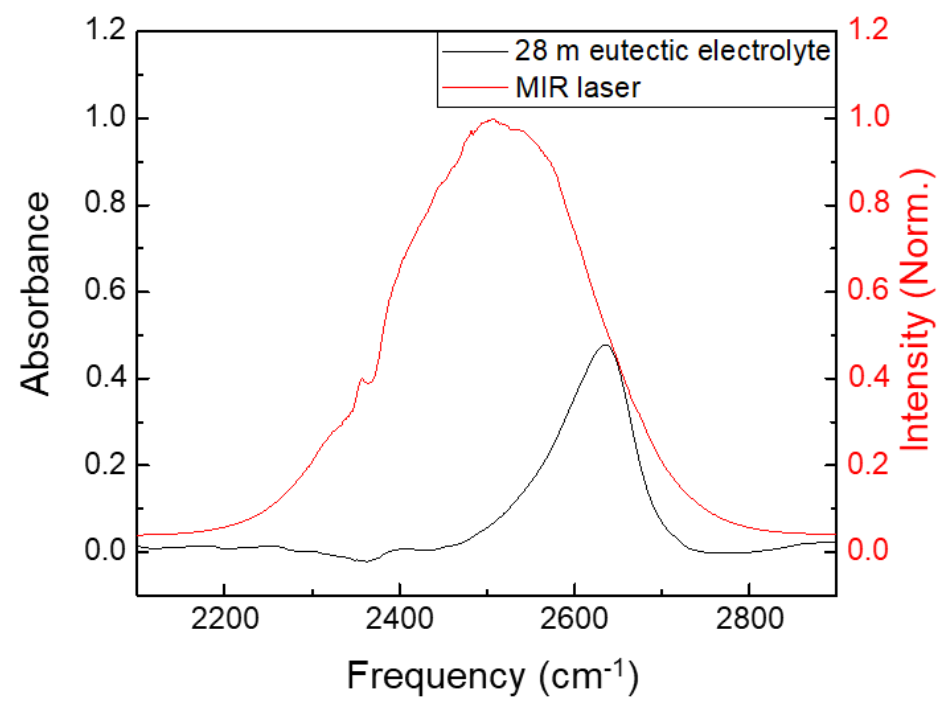

Figure S1. The spectrum of MIR pulse laser and background-subtracted FTIR spectrum of the OD stretching band in 28 m eutectic electrolyte. MIR pulse is purged with $\mathrm{N}_{2}$ gas to remove the absorption of $\mathrm{CO}_{2}$ in the air.

Dielectric relaxation spectroscopy. Dielectric relaxation was measured by Agilent 85070E Dielectric Probe Kit installed with N5247A PAN-X network analyzer. DRS measures the permanent dipoles of the electrolyte in a time-dependent small-amplitude electric field. The dielectric spectra were recorded at $25^{\circ} \mathrm{C}$ in the frequency range $0.2 \leq v / \mathrm{GHz} \leq 67$. The dielectric response to the oscillating electric field can be expressed as a complex total permittivity,

$\hat{\eta}(v)=\hat{\varepsilon}(v)-i k /\left(2 \pi v \varepsilon_{0}\right)$,

where $\hat{\varepsilon}(v)$ is the complex permittivity, $k$ is the ionic conductivity of the electrolyte, and $\varepsilon_{0}$ is the permittivity of free space. The complex permittivity is given as

$\hat{\varepsilon}(v)=\varepsilon^{\prime}(v)-i \varepsilon^{\prime \prime}(v)$.

The permittivity, $\varepsilon^{\prime}(v)$, reflects how far the dipoles in the electrolyte respond to the electric field. The dielectric loss, $\varepsilon^{\prime \prime}(v)$, presents the dissipation of the electromagnetic radiation by the electrolyte. The permittivity decreases from the static permittivity (dielectric constant, $\varepsilon_{s}$ ) at low frequency to the so-called infinite-frequency limit at high frequency $\left(\varepsilon_{\infty}\right)$. In the dispersion region, the absorption of electromagnetic energy by the electrolyte gives rise to dielectric loss.

For aqueous electrolyte analysis, the measured DR spectra were fitted using three Debye-type relaxations reflecting the reorientation of bulk-like water, slow water, and ion pairs. 
$\hat{\varepsilon}(\mathrm{v})=\frac{S_{\text {Bulk-like water }}}{1+i 2 \pi v \tau_{\text {Bulk-like water }}}+\frac{S_{\text {Slow water }}}{1+i 2 \pi v \tau_{\text {Slow water }}}+\frac{S_{I P}}{1+i 2 \pi v \tau_{I P}}+\varepsilon_{\infty}$

where $S$ and $\tau$ are the dielectric strength and relaxation time of chemical species in electrolytes (bulk-like water, slow water, and ion pair), respectively. The molar concentration of the electrolyte species $\left(c_{i}\right)$ is related to the dielectric strength $\left(S_{i}\right)$ of relaxation processes, as described by the Cavell equation,

$c_{i}=S_{i} \times \frac{2 \varepsilon_{S}+1}{\varepsilon_{S}} \frac{k_{B} T \varepsilon_{0}}{N_{A} \mu_{i, e f f}^{2}} \approx \frac{c_{0}}{S_{0}} \times S_{i}$

where $N_{A}$ is the Avogadro constant, $k_{B}$ is the Boltzmann constant, $T$ is the temperature, and $\mu_{i, e f f}$ is the effective dipole moment of species $i$. To obtain the molar concentration of bulklike water, we used the approximate expression (where $S_{0} \approx 72.9$ and $c_{0} \approx 55.4 \mathrm{~mol} / \mathrm{L}$ are the dielectric strengths and $\mathrm{H}_{2} \mathrm{O}$ concentrations of the pure water).

PFG-NMR. The PFG-NMR measurements were performed for $0.4 \mathrm{~mL}$ non-deuterated solvent in a standard $5 \mathrm{~mm}$ NMR tube without field/frequency locking at $298 \pm 0.2 \mathrm{~K}$. A smaller NMR tube was inserted into a standard $5 \mathrm{~mm}$ NMR tube with $0.15 \mathrm{~mL} \mathrm{D}_{2} \mathrm{O}$. The spectra were measured by a Bruker Avance III $400(400.13 \mathrm{MHz})$ spectrometer. The gradient strength of the machine was calibrated using a reference sample with a known self-diffusion coefficient.

For each measurement, two dummy scans and 16 scans were recorded with a relaxation delay of $2 \mathrm{~s}$. The length of the gradient pulse was optimized for every nucleus in each sample, which was 4.0-6.0 ms for ${ }^{1} \mathrm{H}-, 14.0-20.0 \mathrm{~ms}$ for ${ }^{7} \mathrm{Li}$ - and 5.6-9.4 ms for ${ }^{19} \mathrm{~F}-\mathrm{DOSY}$ s. A linear gradient ramp with 12 increments for ${ }^{1} \mathrm{H},{ }^{7} \mathrm{Li}$, and ${ }^{19} \mathrm{~F}$ between 5 and $95 \%$ of the maximum gradient strength was applied for the diffusion-relevant gradients. The spectra were processed through the Bruker program Topspin ${ }^{\circledR}$, and the self-diffusion coefficients were obtained with the Bruker software T1/T2 package.

Measurement of viscosity. To measure the viscosity of electrolytes, we used a sine-wave vibro viscometer SV-10 (A\&D Company, Japan) with the range of measurement from $0.3 \mathrm{mPa} s$ to $10,000 \mathrm{mPa}$ s. Before the formal measurement of electrolyte viscosity, the SV-10 viscometer was calibrated with deionized water. Each measurement was performed two times and the average value was adopted as the final value. 
Supplemental Note 2. Peak assignment of two water species in FTIR spectroscopy

a

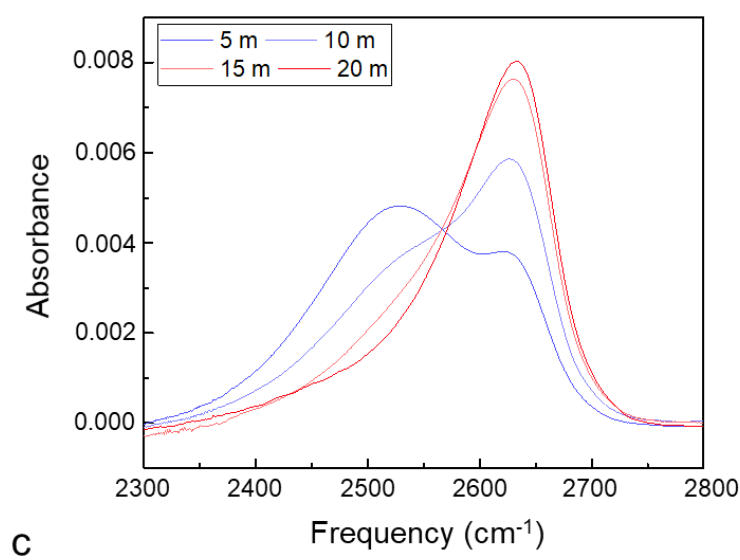

$\mathrm{C}$

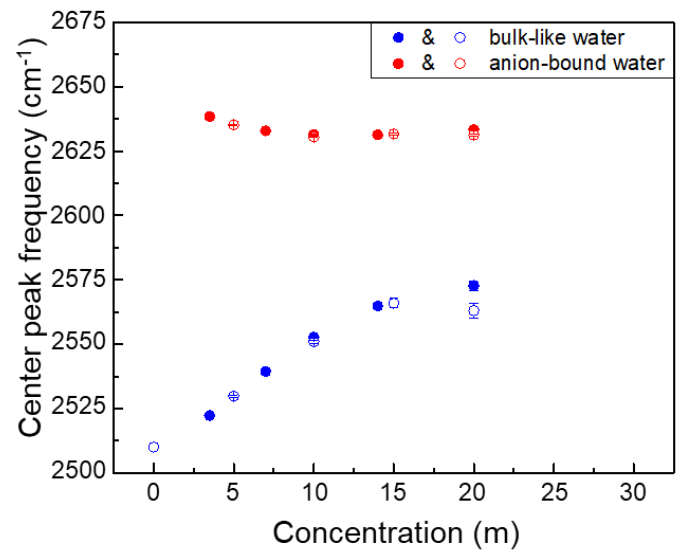

b
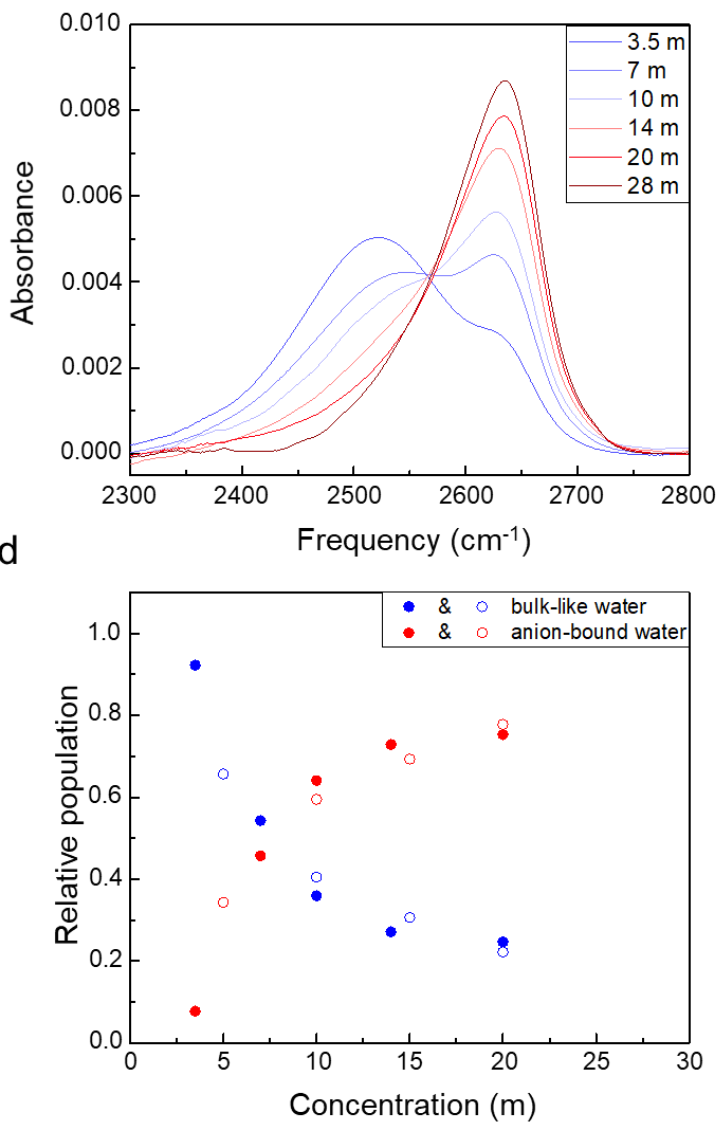

Figure S2. FTIR analysis of OD stretching band in LiTFSI \& eutectic electrolytes in 5 v\% HOD solutions. a) and b) are background-subtracted FTIR spectra of the OD stretch mode in LiTFSI and eutectic solutions, respectively. Center peak frequencies c) and relative population d) of bulk-like water and anion-bound water in LiTFSI and eutectic solutions with various concentrations. For comparison, closed \& open symbols represent the value obtained by eutectic and LiTFSI electrolytes, respectively. Due to the significant blue shift and reduced intensity of the bulk-like water peak in $28 \mathrm{~m}$ eutectic solutions, we could not determine the two water species in FTIR spectra.

The FTIR spectra of the OD stretching mode in Fig.S1 showed two bands at 2510 and 2630 $\mathrm{cm}^{-1}$. As the lithium salt concentration increases, the intensity of the high-frequency band gradually increases, whereas that at the low-frequency decreases. This suggests that there are two components in the OD stretching band in eutectic electrolytes, as in the case of LiTFSI electrolytes. To quantitatively analyze the OD stretching band, two Gaussian functions were used. Previous two-dimensional IR (2D-IR) spectroscopy study on LiTFSI electrolytes revealed that the IR peak at $\sim 2630 \mathrm{~cm}^{-1}$ corresponds to the water (HOD) molecules interacting with the sulfonyl group $(\mathrm{S}=\mathrm{O})$ of TFSI anions. ${ }^{2}$ Furthermore, recent MD simulation studies of WiSE showed that most water molecules interact only with the oxygen atoms of the TFSI sulfonyl groups, other water molecules, and Li-ions. ${ }^{3}$ Considering the chemical structures of two anions (Fig.1 a), we assign the OD absorption band at $2630 \mathrm{~cm}^{-1}$ to the anion-bound water $\left(\mathrm{OD} \cdots \mathrm{O}=\mathrm{S}\right.$ of $\mathrm{TFSI}^{-}$or BETI ${ }^{-}$) and that at $\sim 2510 \mathrm{~cm}^{-1}$ to the bulk-like (water-H-bonded) water 
$\left(\mathrm{OD} \cdots \mathrm{H}_{2} \mathrm{O}\right) .^{2}$ In addition to the interaction of OD group with anions, the vibrational properties, e.g., OD stretch frequency and transition dipole moment, could be affected by the interaction of OD group with lithium cations. However, it is known that the vibrational frequency of the OD stretch mode of HDO in aqueous electrolytes is more substantially shifted when the D atom of HDO interacts with neighboring anions than the case when the $\mathrm{O}$ atom of HDO interacts with cations. The Bakker group showed that the nature of cation hardly affects the OD stretching band of HDO in salt-concentrated solutions. ${ }^{4}$ Therefore, it is challenging to identify and separately investigate the effect of the water interaction with $\mathrm{Li}^{+}$by merely examining the frequency shift of the corresponding band in the experimentally measured IR spectrum. To obtain accurate populations of the two distinctive water species, the frequency-dependence of vibrational transition dipole moment (i.e., non-Codon effect) which can be obtained from IR/Raman ratio was considered (Fig.S3). ${ }^{5}$ The OD stretching frequencies of the anion-bound and the bulk-like water molecules in the eutectic system are almost identical to those in the LiTFSI electrolytes as shown in Fig.S1, which indicates that water mainly interacts with the sulfonyl groups, other water molecules, and Li-ions. In other words, the OD stretching frequency does not reflect the water structure change induced by the added $-\mathrm{CF}_{2}$ - group in the

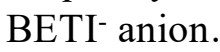




\section{Supplemental Note 3. MD simulation and analysis methods}

Computational model and simulation details. For molecular dynamics (MD) simulation of the aqueous solutions of LiTFSI, we have taken the force field model developed in a previous study based on the Amber force field. ${ }^{3}$ For the simulation of the eutectic mixture of LiTFSI and LiBETI, we applied the same modeling procedure used for LiTFSI to develop a new model for the BETI anion, which is different from TFSI by an extra $\mathrm{CF}_{2}$ group on each side of the sulfonimide group. We have found that the reproduction of experimental self-diffusion coefficients of water, Li-ion, and TFSI and BETI anions in the $28 \mathrm{~m}$ aqueous solutions of the eutectic mixture $\mathrm{Li}(\mathrm{TFSI})_{0.7}(\mathrm{BETI})_{0.3}$ is possible by uniform scaling of ionic partial charges by the factor of 0.76 . This scaling factor is $5 \%$ smaller than the factor of 0.8 used in the LiTFSI solutions and the resulting self-diffusion coefficients were within 15\% (for Li-ions, TFSI and BETI anions) or $30 \%$ (for water molecules) of the experimental data. Details of this result will be reported elsewhere. The Lennard-Jones (LJ) parameters of the BETI anion were taken from the model of TFSI. ${ }^{3}$ New force field parameters used for the eutectic solutions are given in Table S1 and S2 below.

Table S1. Intramolecular force field parameters of the BETI anion. Functional forms of the force field and parameters not listed here can be found in Ref. ${ }^{3}$ Subscripts $m$ and $t$ refer to $\mathrm{CF}_{2}$ and $\mathrm{CF}_{3}$ groups, respectively.

\begin{tabular}{|c|c|c|c|c|c|}
\hline Bond & $r_{0}(\AA)$ & $k_{b}\left(\mathrm{kcal} \mathrm{mol}^{-1} \AA^{-2}\right)$ & Bond & $r_{0}(\AA)$ & $k_{b}\left(\mathrm{kcal} \mathrm{mol}^{-1} \AA^{-2}\right)$ \\
\hline $\mathrm{S}-\mathrm{C}_{\mathrm{m}}$ & 1.8726 & 113.6001 & $\mathrm{C}_{\mathrm{m}}-\mathrm{C}_{\mathrm{t}}$ & 1.5380 & 222.6112 \\
\hline Angle & $\theta_{0}(\operatorname{deg})$ & $k_{\theta}\left(\mathrm{kcal} \mathrm{mol}^{-1} \mathrm{rad}^{-2}\right)$ & Angle & $\theta_{0}(\operatorname{deg})$ & $k_{\theta}\left(\mathrm{kcal} \mathrm{mol}^{-1} \mathrm{rad}^{-2}\right)$ \\
\hline $\mathrm{F}_{\mathrm{m}}-\mathrm{C}_{\mathrm{m}}-\mathrm{F}_{\mathrm{m}}$ & 107.6185 & 107.7703 & $\mathrm{O}-\mathrm{S}-\mathrm{C}_{\mathrm{m}}$ & 104.8330 & 54.4673 \\
\hline $\mathrm{O}-\mathrm{S}-\mathrm{O}$ & 120.0352 & 91.3346 & $\mathrm{~S}-\mathrm{C}_{\mathrm{m}}-\mathrm{F}_{\mathrm{m}}$ & 111.3537 & 52.7982 \\
\hline $\mathrm{N}-\mathrm{S}-\mathrm{O}$ & 112.3071 & 95.0408 & $\mathrm{~N}-\mathrm{S}-\mathrm{C}_{\mathrm{m}}$ & 101.9732 & 75.9787 \\
\hline S-N-S & 126.1564 & 58.8203 & $\mathrm{~S}-\mathrm{C}_{\mathrm{m}}-\mathrm{C}_{\mathrm{t}}$ & 113.5400 & 81.8993 \\
\hline $\mathrm{F}_{\mathrm{t}}-\mathrm{C}_{\mathrm{t}}-\mathrm{F}_{\mathrm{t}}$ & 107.6185 & 98.9041 & $\mathrm{~F}_{\mathrm{m}}-\mathrm{C}_{\mathrm{m}}-\mathrm{C}_{\mathrm{t}}$ & 108.0800 & 85.1764 \\
\hline $\mathrm{C}_{\mathrm{m}}-\mathrm{C}_{\mathrm{t}}-\mathrm{F}_{\mathrm{t}}$ & 110.1300 & 66.0958 & & & \\
\hline Dihedral $^{a}$ & $n$ & $V_{n}(\mathrm{kcal} / \mathrm{mol})$ & Dihedral $^{a}$ & $n$ & $V_{n}(\mathrm{kcal} / \mathrm{mol})$ \\
\hline \multirow[t]{3}{*}{$\mathrm{O}-\mathrm{S}-\mathrm{C}_{\mathrm{m}}-\mathrm{F}_{\mathrm{m}}$} & 1 & -1.38758 & $\mathrm{~N}-\mathrm{S}-\mathrm{C}_{\mathrm{m}}-\mathrm{F}_{\mathrm{m}}$ & 1 & 0.67430 \\
\hline & 2 & -0.37775 & & 2 & 1.26335 \\
\hline & 3 & -0.05297 & & 3 & -0.61934 \\
\hline \multirow[t]{5}{*}{$\mathrm{O}-\mathrm{S}-\mathrm{N}-\mathrm{S}$} & 1 & 5.36204 & $\mathrm{~S}-\mathrm{N}-\mathrm{S}-\mathrm{C}_{\mathrm{m}}$ & 1 & 0.04060 \\
\hline & 2 & -2.77266 & & 2 & -0.20029 \\
\hline & 3 & 0.63208 & & 3 & 0.27341 \\
\hline & 4 & -0.04432 & & 4 & 0.05302 \\
\hline & 5 & 0.54349 & & 5 & 0.12163 \\
\hline \multirow[t]{2}{*}{$\mathrm{F}_{\mathrm{m}}-\mathrm{C}_{\mathrm{m}}-\mathrm{C}_{\mathrm{t}}-\mathrm{F}_{\mathrm{t}}$} & 1 & -0.05320 & $\mathrm{~S}-\mathrm{C}_{\mathrm{m}}-\mathrm{C}_{\mathrm{t}}-\mathrm{F}_{\mathrm{t}}$ & 1 & -0.53051 \\
\hline & 2 & -0.20007 & & 2 & 0.07578 \\
\hline
\end{tabular}




\begin{tabular}{rllll} 
& 3 & -0.15851 & 3 & 0.38912 \\
O-S-C $_{m}-C_{t}$ & 1 & -0.29742 & N-S-C ${ }_{m}-C_{t}$ & 1 \\
& 2 & -0.12693 & 2 & 0.64467 \\
& 3 & 1.14852 & 3 & 1.17277 \\
4 & 0.33556 & 4 & -0.74514 \\
5 & -0.35669 & 5 & -0.06713 \\
\hline
\end{tabular}

$a$. The phase $\gamma$ is set to zero in all cases. These dihedral parameters must be used with complete suppression of the corresponding 1-4 non-bonded interactions.

Table S2. Partial charges of all ionic species in the aqueous solutions of eutectic mixture $\mathrm{Li}(\mathrm{TFSI})_{0.7}(\mathrm{BETI})_{0.3}$. Functional forms of the force field and parameters not listed here can be found in Ref. ${ }^{3}$ Subscripts $\mathrm{m}$ and $\mathrm{t}$ refer to $\mathrm{CF}_{2}$ and $\mathrm{CF}_{3}$ groups, respectively. $\mathrm{T}$ and $\mathrm{B}$ denote TFSI and BETI anions, respectively.

\begin{tabular}{lccccccc}
\hline Atom & $\mathrm{N}(\mathrm{T})$ & $\mathrm{S}(\mathrm{T})$ & $\mathrm{O}(\mathrm{T})$ & $\mathrm{C}(\mathrm{T})$ & $\mathrm{F}(\mathrm{T})$ & $\mathrm{Li}$ & \\
\hline$q(\mathrm{e})$ & -0.41588 & 0.59257 & -0.36754 & 0.20505 & -0.07820 & 0.76000 & \\
\hline Atom & $\mathrm{N}(\mathrm{B})$ & $\mathrm{S}(\mathrm{B})$ & $\mathrm{O}(\mathrm{B})$ & $\mathrm{C}_{\mathrm{m}}(\mathrm{B})$ & $\mathrm{C}_{\mathrm{t}}(\mathrm{B})$ & $\mathrm{F}_{\mathrm{m}}(\mathrm{B})$ & $\mathrm{F}_{\mathrm{t}}(\mathrm{B})$ \\
\hline$q(\mathrm{e})$ & -0.37758 & 0.59409 & -0.35965 & 0.01130 & 0.33857 & -0.06524 & -0.09513 \\
\hline
\end{tabular}

We have performed MD simulations of aqueous solutions of LiTFSI at 1, 5, 10, 15, and $21 \mathrm{~m}$ and of an aqueous solution of the eutectic mixture Li(TFSI) $)_{0.7}(\mathrm{BETI})_{0.3}$ at $28 \mathrm{~m}$. Each system contains 1000 TIP4P-Ew $\mathrm{H}_{2} \mathrm{O}$ molecules and different numbers of ionic species corresponding to the given concentration. For example, the eutectic solution at $28 \mathrm{~m}$ contains $504 \mathrm{Li}$-ions, 353 TFSI anions, and 151 BETI anions. In the simulation, periodic boundary conditions were applied, and the particle mesh Ewald method was used to calculate Coulombic interaction with a cutoff radius of $10 \AA$ for the LJ and screened real-space Coulombic interactions. Time step size was 1 fs. For equilibration of each system, MD simulations were performed first in the NPT ensemble for more than $100 \mathrm{~ns}$, then in the NVT ensemble for $10 \mathrm{~ns}$. From this, a production simulation was performed for $61 \mathrm{~ns}$ in the NVE ensemble. The CPU version of the AMBER pmemd program was used in the production run to ensure energy conservation.

The classification of $\mathrm{OH}$ groups into water-bound and anion-bound types are made as follows: (i) All $\mathrm{OH}$ groups are checked for intermolecular contact $\left(\mathrm{H}_{\mathrm{W}}-\mathrm{O}_{\mathrm{W}}\right.$ and $\left.\mathrm{H}_{\mathrm{W}}-\mathrm{O}_{\mathrm{T}}\right)$ at $0.1 \mathrm{ps}$ intervals for $2 \mathrm{ps}$, (ii) if a given type of contact is observed at $40 \%$ or more frames during the 2 ps period regardless of the identities of the $\mathrm{O}_{\mathrm{W}}$ or $\mathrm{O}_{\mathrm{T}}$ atoms, the $\mathrm{OH}$ group is selected in that 2 ps time period, (iii) repeat this ten times and only the $\mathrm{OH}$ groups that are selected in ten consecutive 2 ps periods are used for analysis. Note that this selection method does not check for the identity of the surrounding $\mathrm{O}_{\mathrm{W}}$ or $\mathrm{O}_{\mathrm{T}}$ atoms, and therefore the exchange of oxygen atoms of the same type is allowed.

Computational study. In a previous computational study of the aqueous solutions of LiTFSI, 
we have established that there are only four types of strong electrostatic interactions (determined by the presence of the first peak within $2.5 \AA$ in the radial distribution function (RDF)): $\mathrm{Li} \cdots \mathrm{O}_{\mathrm{T}}, \mathrm{Li} \cdots \mathrm{O}_{\mathrm{W}}, \mathrm{H}_{\mathrm{W}} \cdots \mathrm{O}_{\mathrm{T}}$ and $\mathrm{H}_{\mathrm{W}} \cdots \mathrm{O}_{\mathrm{W}}$ where subscripts $\mathrm{W}$ and $\mathrm{T}$ denote water molecules and TFSI- anions, respectively. Extending this notion, we first studied the local solvation environments of those four atoms $\left(\mathrm{Li}, \mathrm{O}_{\mathrm{A}}, \mathrm{O}_{\mathrm{W}}, \mathrm{H}_{\mathrm{W}}\right), \mathrm{OH}$ groups, and water molecules in the $28-\mathrm{m}$ eutectic solution by counting surrounding atoms within a cutoff distance $r_{\mathrm{c}}$ from each atom of interest. Here, we do not distinguish sulfonyl oxygens of TFSI and BETI anions for simplicity and treat them as a single type denoted as $\mathrm{O}_{\mathrm{A}}$, and $r_{\mathrm{c}}$ was taken as the location of the first minimum in the RDF of each atom pair. From this analysis, we could obtain the distribution of the solvation environment of atoms, $\mathrm{OH}$ groups, and water molecules, characterized by the atomic composition in the first solvation shell (Table S3). From this, we first note that $97 \%$ of all $\mathrm{O}_{\mathrm{W}}$ (and therefore water molecules) coordinate one or two Li-ions, leaving only $3 \%$ without contact with Li-ions. We also note that $35 \%$ of $\mathrm{H}_{\mathrm{W}}$ atoms exist as "free" without making an H-bond. This is a significant increase from $8.2 \%$ in the 1 -m LiTFSI solution and indicates a significant disruption of the $\mathrm{H}$-bond network at $28 \mathrm{~m}$. Adding up the $\mathrm{H}_{\mathrm{W}}$ populations with $n_{\mathrm{OW}}>0$ or $n_{\mathrm{OT}}>0$, we can also see that $10 \%$ of $\mathrm{OH}$ groups are exclusively water-bound, 54\% are exclusively anion-bound, and virtually no $\mathrm{OH}$ groups make an H-bond as a donor with another water and an anion simultaneously. Including the free $\mathrm{OH}$ groups, we could therefore identify three types of $\mathrm{OH}$ groups of water molecules with a significant population in the $28-\mathrm{m}$ eutectic solution. If we make an as-yet unjustified assumption that the stretch frequencies of the anion-bound and free OD groups are similar to each other and regard the two as a single type corresponding to the experimental high-frequency OD groups, the computationally determined populations agree well with the populations of the bulk-like and anion-bound water obtained from FTIR and IR PP measurements (Fig.1 d). However, it would be necessary to spectroscopically characterize the free $\mathrm{OH} / \mathrm{OD}$ groups to fully resolve this issue. From the distribution of $\mathrm{OH}$ groups in Table $\mathrm{S} 3$, we can see that most of these free $\mathrm{OH}$ groups arise when the $\mathrm{OH}$ group coordinates a Li-ion. This means that water molecules interacting with a Li-ion (97\% at $28 \mathrm{~m}$ according to Table S3) tend to lose at least one $\mathrm{H}$-bond with $\sim 30 \%$ probability, possibly contributing to the mobility of the interaction complexes. From this analysis, we also found that $81 \%$ of all water molecules are in contact with one or more $\mathrm{O}_{\mathrm{T}}$ atoms and $20 \%$ are in contact with one or more $\mathrm{O}_{\mathrm{W}}$ atoms. These numbers include $12 \%$ of water molecules that contact both $\mathrm{O}_{\mathrm{T}}$ and $\mathrm{O}_{\mathrm{W}}$ atoms. Finally, $12 \%$ of water molecules do not interact with either $\mathrm{O}_{\mathrm{T}}$ or $\mathrm{O}_{\mathrm{W}}$. This result reminds us that the spectroscopic classification of water molecules by the OD stretch mode as presented above applies to individual OD groups, not the water molecules.

To study the rotational dynamics of water molecules and to compare them with the anisotropic IR PP results, we have calculated the rotational time correlation function (TCF) corresponding to the IR-PP polarization anisotropy $\mathrm{r}(t)$ as follows ${ }^{6}$

Table S3. Distribution of atomic composition in the first solvation shells of atoms, OH groups, and water molecules in the $28 \mathrm{~m}$ aqueous solutions of $\mathrm{Li}(\mathrm{TFSI})_{0.7}(\mathrm{BETI})_{0.3}$ as determined by MD simulation. Atomic compositions with a relative population larger than $1 \%\left(\mathrm{O}_{\mathrm{W}}, \mathrm{H}_{\mathrm{W}}\right)$ or 
$2 \%(\mathrm{OH}$ groups, water molecules) are listed.

\begin{tabular}{ll|ll}
\hline $\mathrm{O}_{\mathrm{W}}$ & & $\mathrm{H}_{\mathrm{W}}$ & \\
\hline$n_{\mathrm{HW}}: n_{\mathrm{Li}}$ & Population & $n_{\mathrm{OW}}: n_{\mathrm{OA}}$ & Population \\
\hline $0: 1$ & 0.731 & $0: 0$ & 0.349 \\
$0: 2$ & 0.073 & $0: 1$ & 0.519 \\
$1: 0$ & 0.011 & $0: 2$ & 0.025 \\
$1: 1$ & 0.167 & $1: 0$ & 0.098 \\
$2: 0$ & 0.016 & Rest & 0.009 \\
Rest & 0.002 & & \\
\hline OH groups & & Water molecules & \\
\hline$n_{\mathrm{OW}}: n_{\mathrm{OA}}: n_{\mathrm{HW}}: n_{\mathrm{Li}}$ & Population & $n_{\mathrm{OW}}: n_{\mathrm{OA}}: n_{\mathrm{HW}}: n_{\mathrm{Li}}$ & Population \\
\hline $0: 1: 0: 1$ & 0.359 & $0: 1: 0: 1$ & 0.291 \\
$0: 0: 0: 1$ & 0.280 & $0: 2: 0: 1$ & 0.179 \\
$0: 1: 1: 1$ & 0.100 & $0: 0: 0: 1$ & 0.098 \\
$1: 0: 0: 1$ & 0.073 & $1: 1: 0: 1$ & 0.077 \\
$0: 1: 0: 2$ & 0.046 & $0: 2: 1: 1$ & 0.063 \\
$0: 0: 1: 1$ & 0.044 & $0: 1: 1: 1$ & 0.056 \\
Rest & 0.098 & $1: 0: 0: 1$ & 0.055 \\
& & $0: 2: 0: 2$ & 0.031 \\
& & Rest & 0.150 \\
\hline
\end{tabular}

$\mathrm{r}(t)=\left(\frac{2}{5}\right)\left\langle P_{2}\left(\widehat{\boldsymbol{u}}_{O H}(t) \cdot \widehat{\boldsymbol{u}}_{O H}(0)\right)\right\rangle$

where $\widehat{\boldsymbol{u}}_{O H}(t)=\boldsymbol{r}_{O H}(t) /\left|\boldsymbol{r}_{O H}(t)\right|$ is the unit vector in the direction of an $\mathrm{OH}$ bond and $P_{2}(x)=\left(3 x^{2}-1\right) / 2$. The rotational TCF is calculated for the LiTFSI solutions from 1 to 21 m concentrations and a 28 m eutectic solution of $\mathrm{Li}(\mathrm{TFSI})_{0.7}(\mathrm{BETI})_{0.3}$, and the results are shown in Fig.S2. To enable direct comparison with the experimental IR-PP anisotropy decay measurements, we have selected only those $\mathrm{OH}$ groups predominantly bound to $\mathrm{O}_{\mathrm{W}}$ (waterbound $\mathrm{OH}$ ) or $\mathrm{O}_{\mathrm{T}}$ (anion-bound $\mathrm{OH}$ ) over a series of 20 ps periods and calculated the TCF separately for each type of $\mathrm{OH}$ groups. From Fig.S3a and Fig.S3b, we note that the rotational TCF displays ultrafast inertial decay at less than $0.2 \mathrm{ps}$, followed by slower multi-exponential decay. Comparing the water-bound (Fig.S3a) and anion-bound (Fig.S3b) OH groups, the TCF of the former varies much more wildly with salt concentration and exhibits a crossover at $\sim 0.3$ ps which is not as noticeable in the latter. However, in both cases, we can see that the shorttime decay becomes faster, but the long-time decay becomes slower with increasing salt concentration. This result indicates that the rotation of $\mathrm{OH}$ groups reflects different solvation environments at different time scales: the local solvation structure in the vicinity of the $\mathrm{OH}$ group at short times and the long-range structure and macroscopic viscosity at longer times. 
Biexponential fitting of the rotational TCF presented in Figure S3 are performed using the function $2.5 r(t)=a e^{-t / \tau_{1}}+b e^{-t / \tau_{2}}$, excluding the initial 0.2 ps portion, which corresponds to the inertial decay component. The magnitude of this inertial component is separately estimated using the parameter $f_{\text {iner }}=1-a-b$. From this biexponential fitting analysis of the rotational TCF presented in Table S4 of SI, we can see that the magnitude of the ultrafast inertial decay component increases with concentration and accounts for up to $27 \%$ (water-bound $\mathrm{OH}$ ) or $38 \%$ (anion-bound $\mathrm{OH}$ ) of the total decay. Since this inertial decay is non-exponential, it cannot be captured in the experimentally determined rotational relaxation times plotted in Fig.2c and Fig.2e. Also, the experimental anisotropy decay of the OD groups in HOD molecules is expected to be slower than the computational result obtained with $\mathrm{H}_{2} \mathrm{O}$ molecules. Considering these limitations, the experimental anisotropy decay behavior in Fig.S3 agrees reasonably well with the computational results. In particular, the rotational relaxation of anion-bound $\mathrm{OH}$ groups in Fig. S3 c and 2d show nearly quantitative agreement of the two results
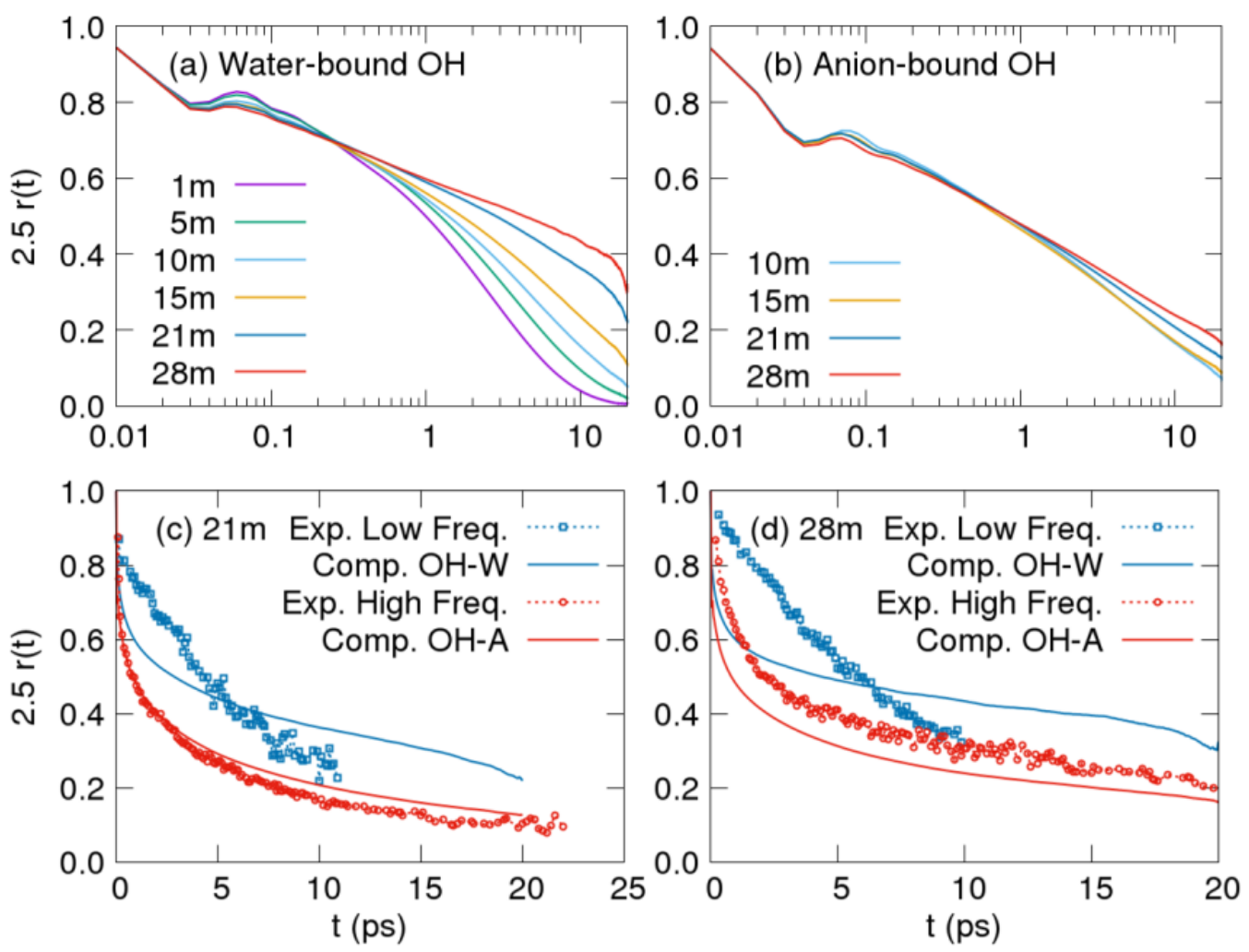

Figure S3. OH-bond rotational dynamics from MD simulations. Rotational TCF of a) water-bound OH groups and $b$ ) anion-bound $\mathrm{OH}$ groups of LiTFSI solutions (1 to $21 \mathrm{~m}$ ) and a eutectic solution of $\mathrm{Li}(\mathrm{TFSI})_{0.7}(\mathrm{BETI})_{0.3}(28$ $\mathrm{m})$. Comparison of rotational TCF and IR-PP anisotropy decays in c) $21 \mathrm{~m}(20 \mathrm{~m}$ for experimental data) LiTFSI solution and d) $28 \mathrm{~m} \mathrm{Li(TFSI})_{0.7}(\mathrm{BETI})_{0.3}$ solution. In Fig. S3c and S3d, OH-W and OH-A mean water-bound and anion-bound $\mathrm{OH}$ groups, respectively, and the experimental low/high-frequency data were obtained respectively in the blue/red frequency bands in Fig. 2 a. 
Table S4. Biexponential fitting result of the rotational TCF

\begin{tabular}{|c|c|c|c|c|c|c|}
\hline Molality (m) & 1 & 5 & 10 & 15 & 21 & 28 \\
\hline \multicolumn{7}{|c|}{ Water-bound $\mathrm{OH}$} \\
\hline$a$ & 0.243 & 0.286 & 0.263 & 0.238 & 0.196 & 0.176 \\
\hline$b$ & 0.523 & 0.458 & 0.463 & 0.479 & 0.534 & 0.557 \\
\hline$\tau_{1}(\mathrm{ps})$ & 1.11 & 1.53 & 1.55 & 1.50 & 1.15 & 0.902 \\
\hline$\tau_{2}(\mathrm{ps})$ & 3.86 & 6.38 & 9.31 & 14.3 & 25.9 & 40.2 \\
\hline$f_{\text {iner }}$ & 0.234 & 0.256 & 0.274 & 0.283 & 0.270 & 0.267 \\
\hline \multicolumn{7}{|c|}{ Anion-bound $\mathrm{OH}$} \\
\hline$a$ & 0.202 & 0.243 & 0.271 & 0.285 & 0.271 & 0.263 \\
\hline$b$ & 0.535 & 0.442 & 0.382 & 0.348 & 0.361 & 0.354 \\
\hline$\tau_{1}(\mathrm{ps})$ & 0.792 & 1.28 & 1.40 & 1.60 & 1.63 & 1.77 \\
\hline$\tau_{2}(\mathrm{ps})$ & 11.8 & 12.9 & 12.2 & 14.4 & 18.4 & 26.2 \\
\hline$f_{\text {iner }}$ & 0.263 & 0.315 & 0.347 & 0.367 & 0.368 & 0.383 \\
\hline
\end{tabular}



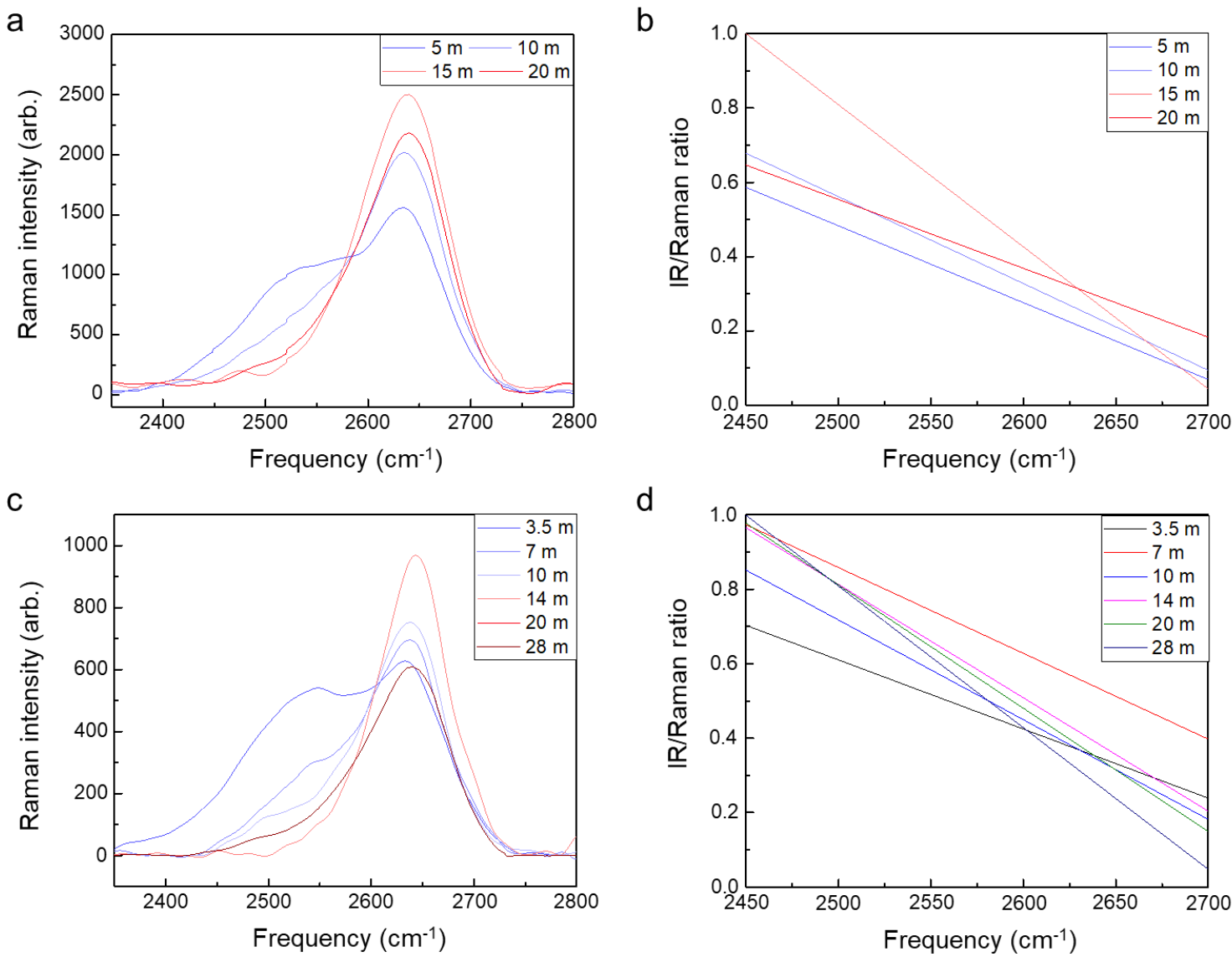

Figure S4. Frequency-dependent transition dipole of various concentrated LiTFSI \& eutectic electrolytes in 5 v\% HOD water solutions. Raman spectrum is calibrated with respect to S-N-S bending mode. a) and c) are Raman intensity of OD stretch mode of various concentrated LiTFSI and eutectic electrolytes in $5 \mathrm{v} \% \mathrm{HOD}$, respectively. b) and d) are the linear fitting line of the IR/Raman ratio. These frequency-dependent transition dipole strengths are used to calibrate the amplitude fraction of FTIR and IR-PP spectrum. 

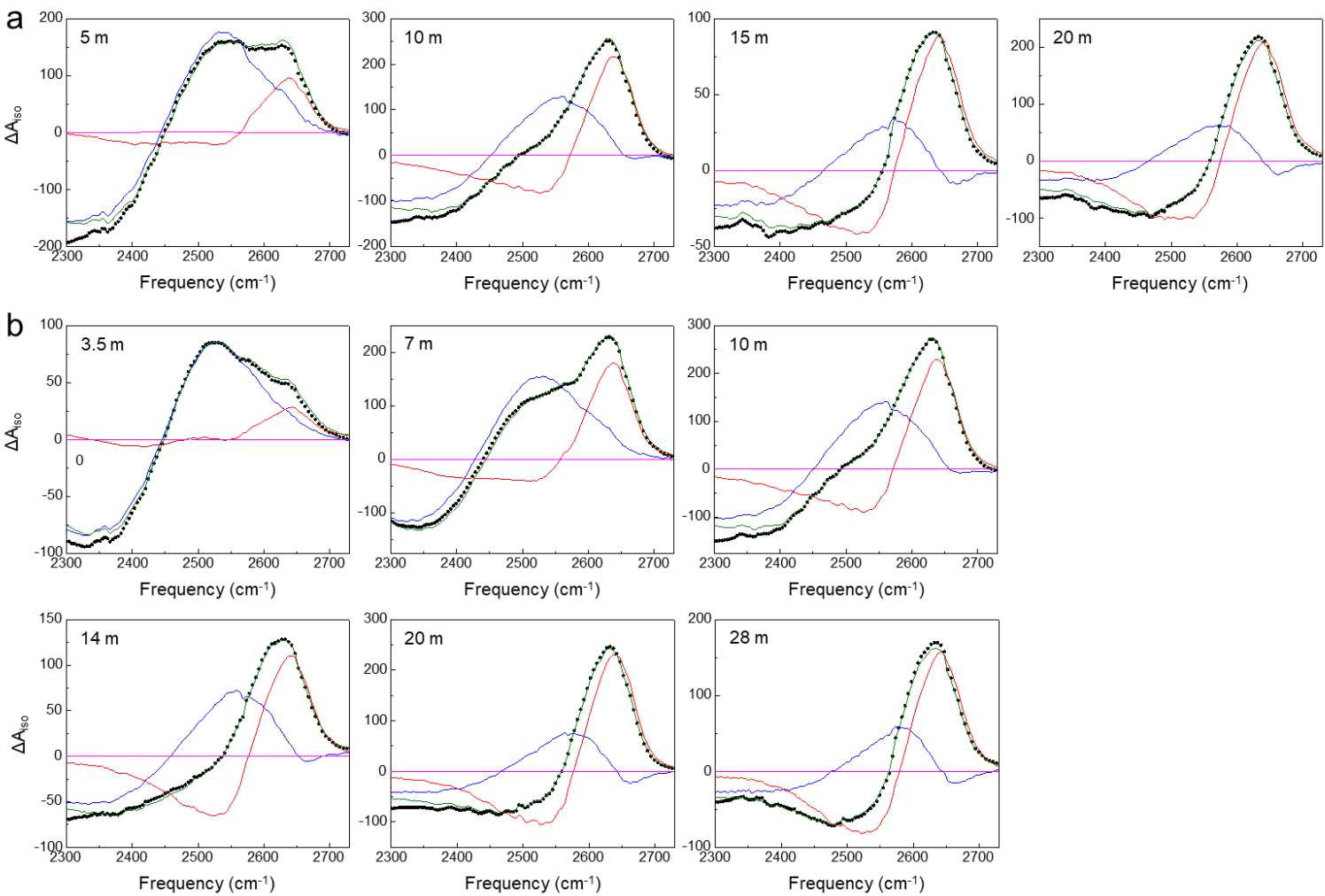

Figure S5. IR-PP spectra of OD stretching band in various concentrated a) LiTFSI and b) eutectic electrolytes where the time delay between the pump and probe pulses is $0.2 \mathrm{ps}$. Closed black squares are isotropic IR-PP data. The blue line corresponds to bulk-like water, whereas the red line corresponds to anion-bound water. The heating contribution to the IR-PP signal is shown in the green line. 


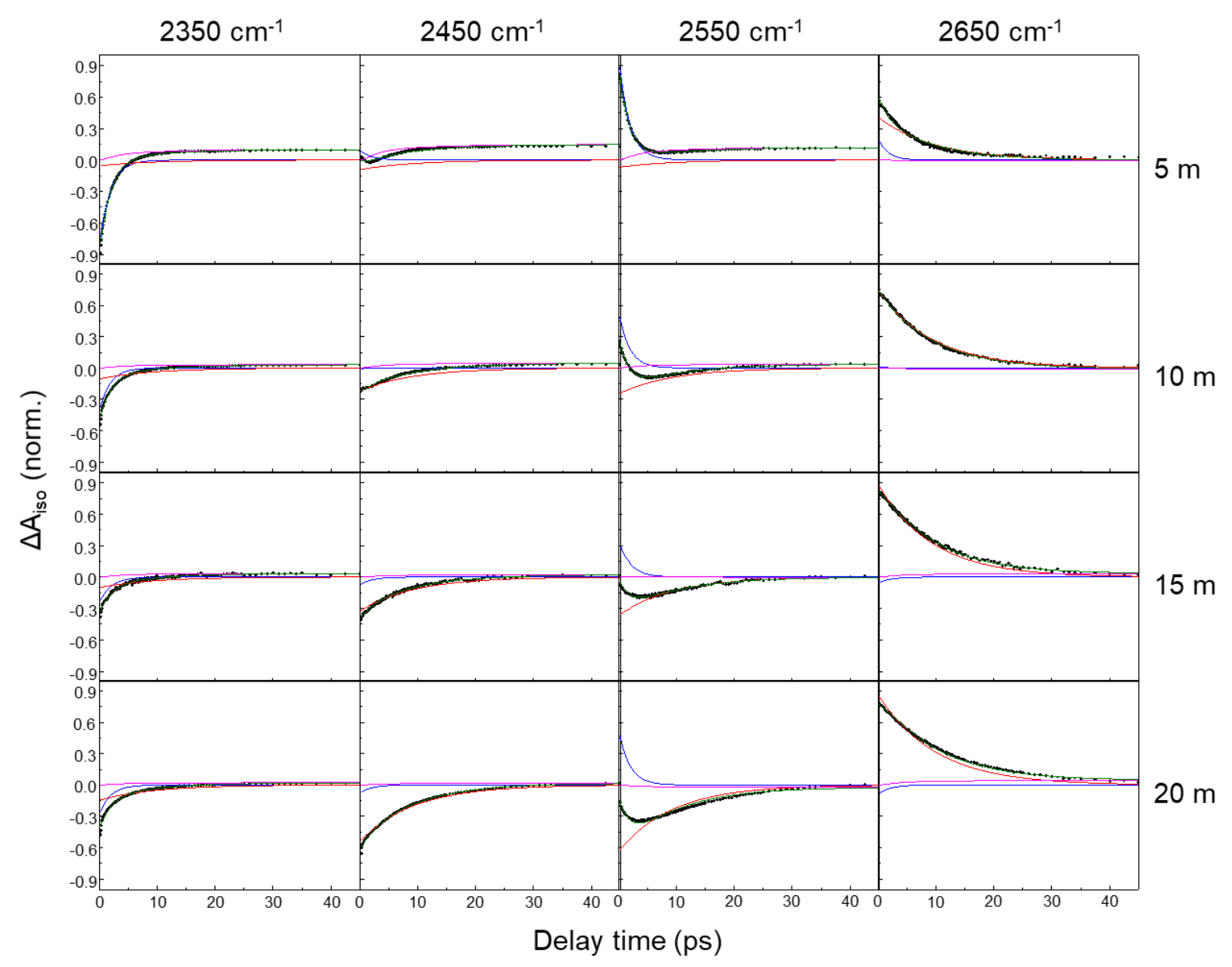

Figure S6. Model fitting analyses of isotropic IR-PP signals at 4 probe frequencies in LiTFSI electrolytes. Closed black squares are isotropic IR-PP data. The blue line corresponds to bulk-like water, whereas the red line corresponds to anion-bound water. The heating contribution to the IR-PP signal is shown in the green line. 


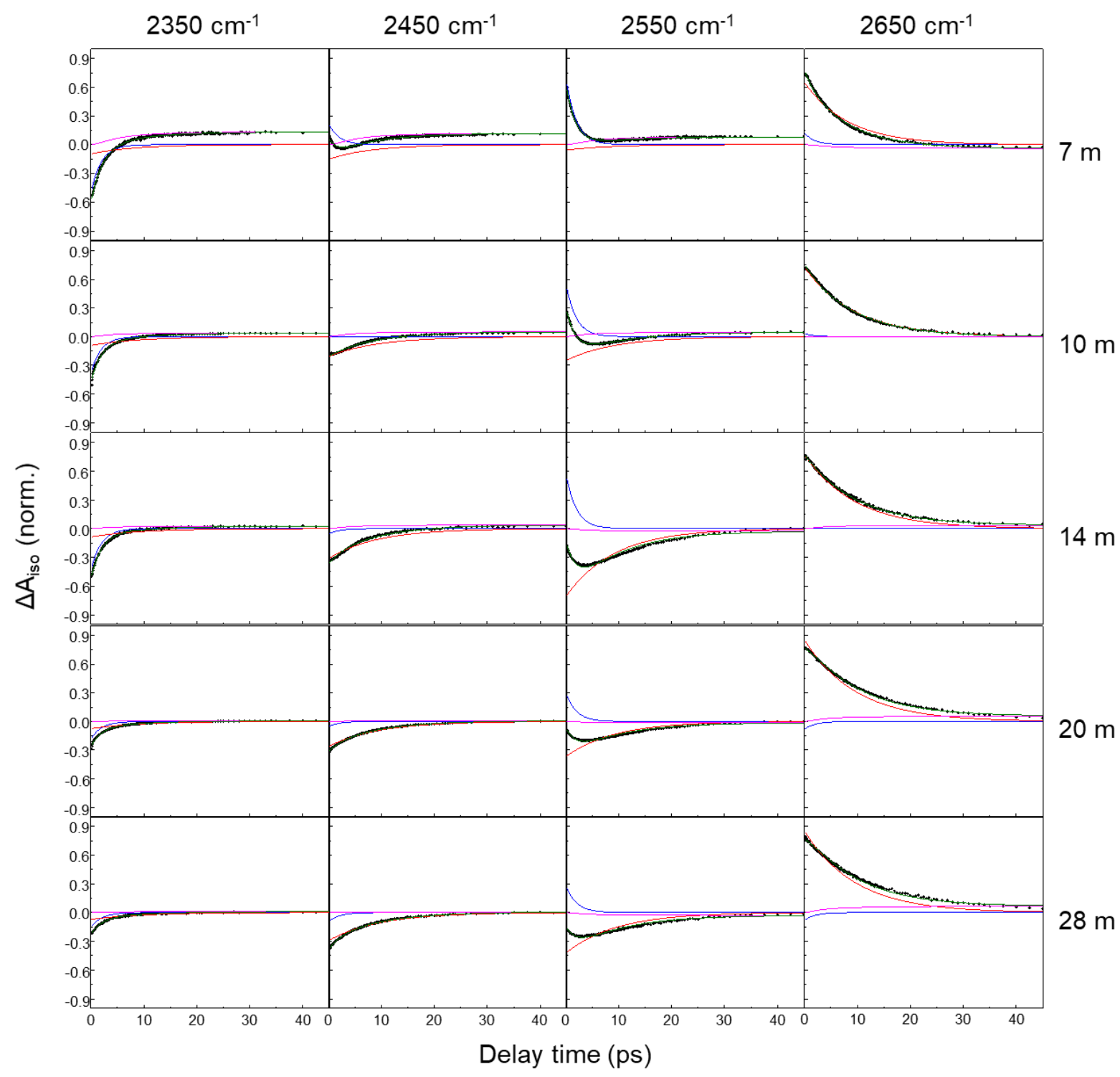

Figure S7. Model fitting analyses of isotropic IR-PP signals at 4 probe frequencies in eutectic electrolytes. Closed black squares are isotropic IR-PP data. The blue line corresponds to bulk-like water, whereas the red line corresponds to anion-bound water. The heating contribution to the IR-PP signal is shown in the green line. 
a

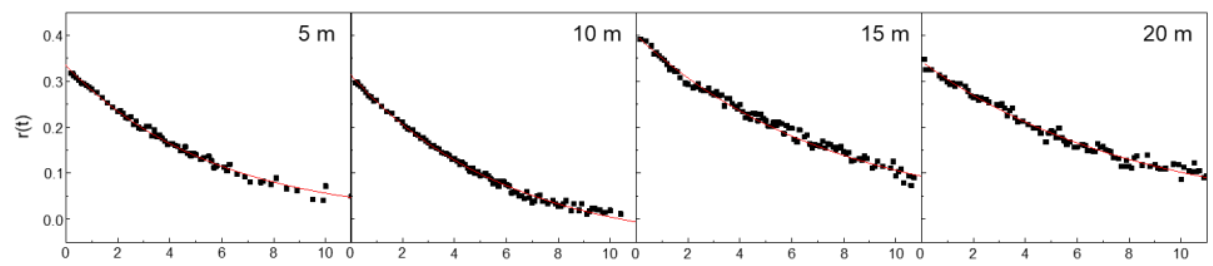

b

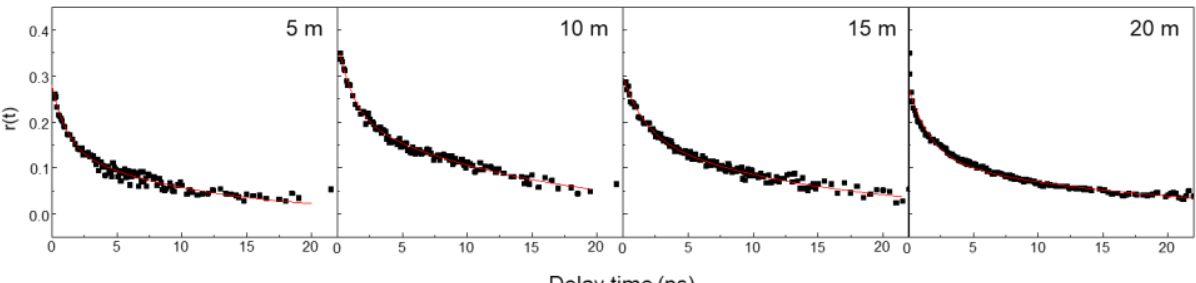

C

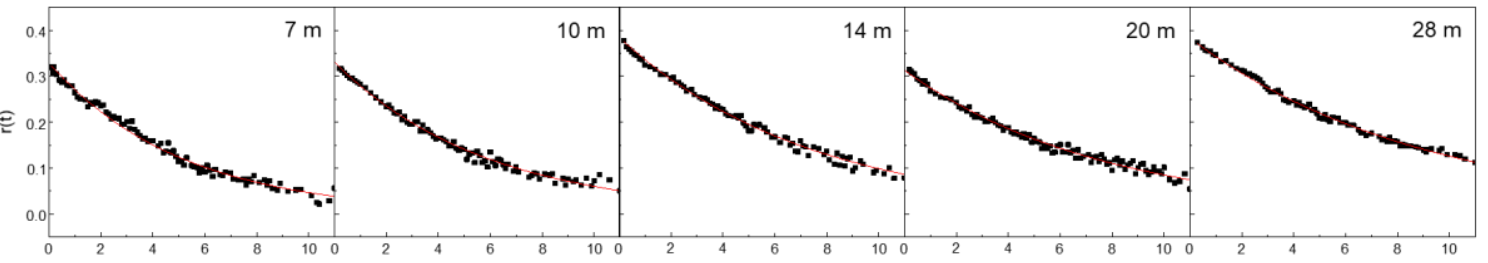

d

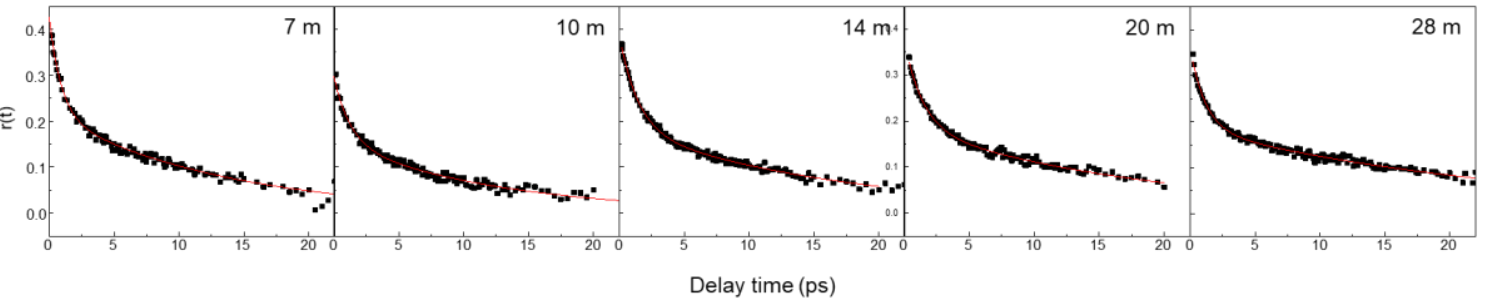

Figure S8. Anisotropic signal decay pattern of various concentrated aqueous electrolytes. a) and c) are an anisotropic signal of bulk-like water (obtained from $2334 \sim 2350 \mathrm{~cm}^{-1}$ ) in LiTFSI and eutectic electrolytes, respectively. b) and d) are anisotropic signals of anion-bound water (obtained from 2662 2683 $\mathrm{cm}^{-1}$ ) in LiTFSI and eutectic electrolytes, respectively. 

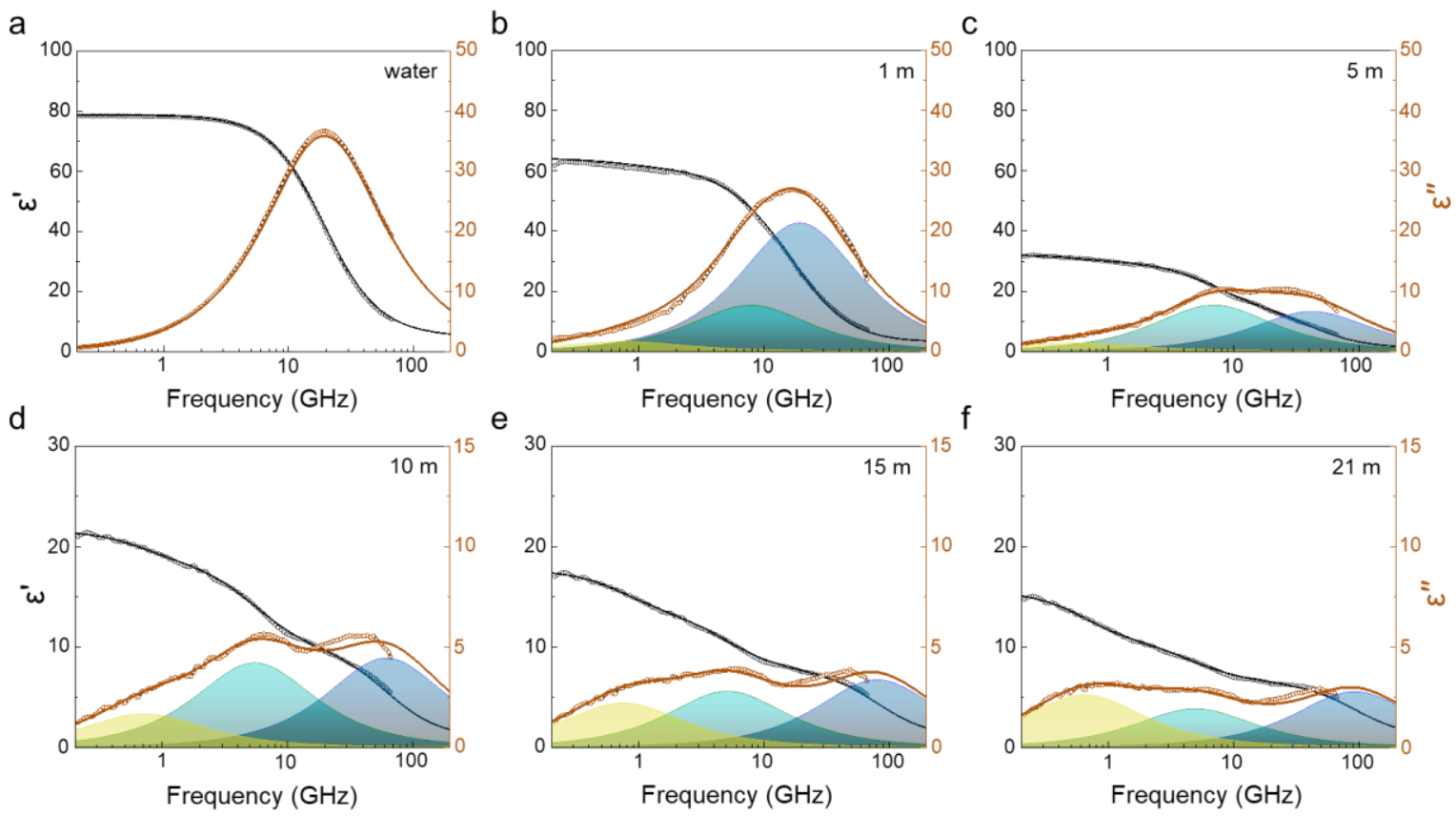

Figure S9. DRS spectra and deconvoluted peaks of water and LiTFSI-water with changing salt concentration (1 $21 \mathrm{~m})$. Relative permittivity, $\varepsilon^{\prime}(v)$, dielectric loss, $\varepsilon^{\prime \prime}(v)$, with the spectrum calculated three Debye processes in LiTFSI-water. The colored areas show the contributions of the ion pair, slow water and bulk-like water relaxation processes.
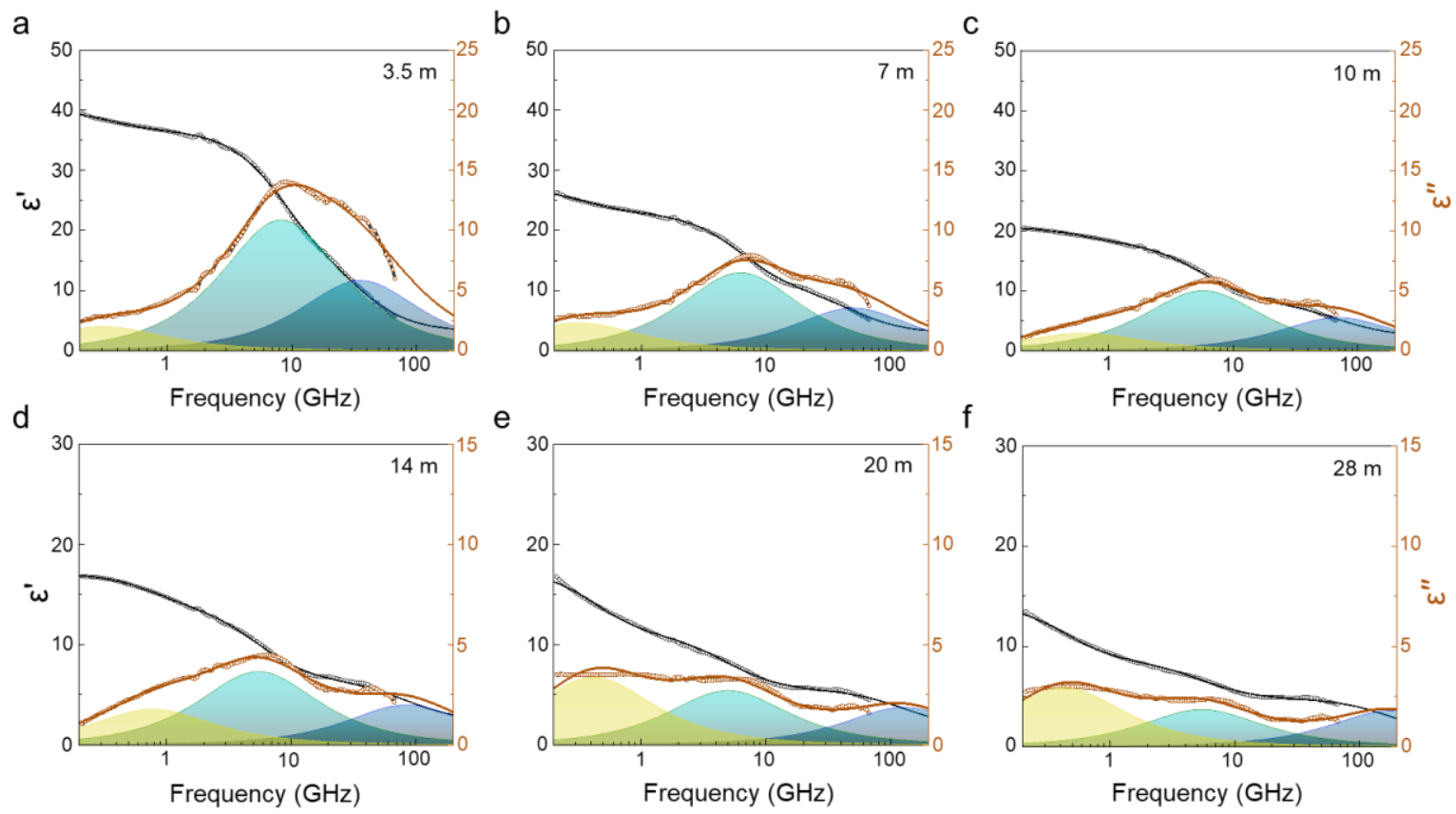

Figure S10. DRS spectra and deconvoluted peaks of Eutectic-water with changing salt concentration (3.5-28 m). Relative permittivity, $\varepsilon^{\prime}(v)$, dielectric loss, $\varepsilon^{\prime \prime}(v)$, with the spectrum calculated three Debye processes in Eutecticwater. The colored areas show the contributions of the ion pair, slow water and bulk-like water relaxation processes. 
a

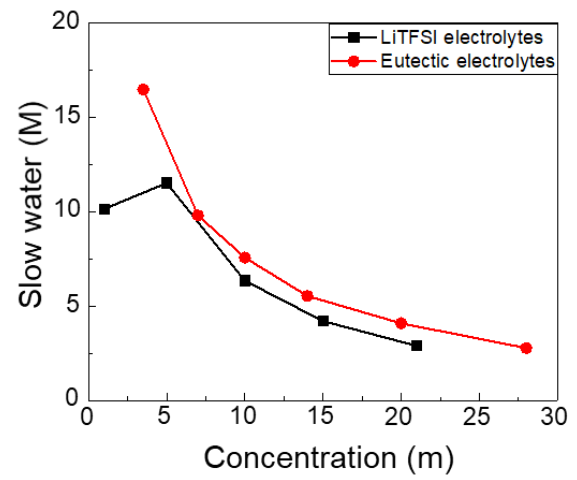

b

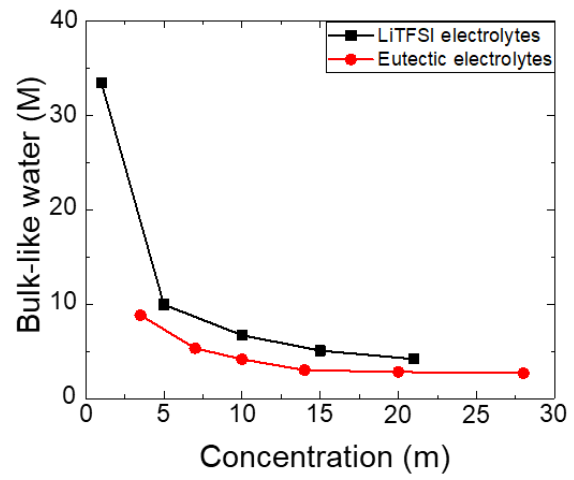

Figure S11. The concentration of slow water and bulk-like water in LiTFSI electrolytes and eutectic electrolytes as a function of salt concentration at $298 \mathrm{~K}$.

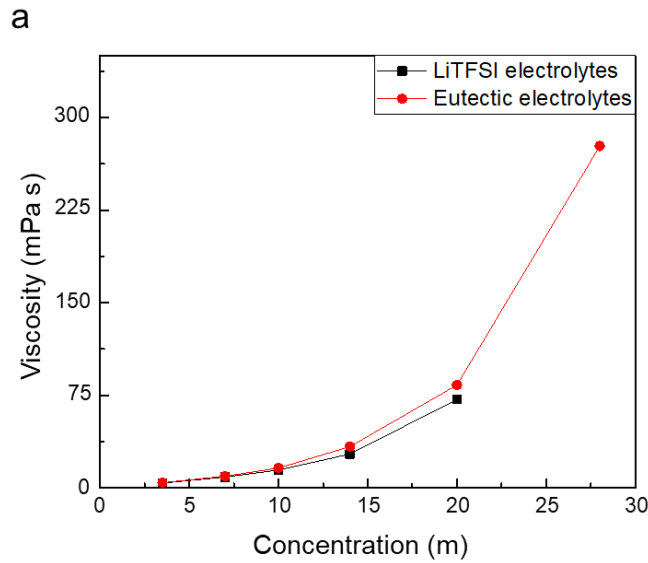

Figure S22. The viscosity of LiTFSI and eutectic electrolytes as a function of salt concentration at $298 \mathrm{~K}$. 
Table S5. Fitted parameters of DRS for LiTFSI electrolytes. Electrical Conductivities, $\boldsymbol{\kappa}$; Static Permittivity, $\boldsymbol{\varepsilon}_{\boldsymbol{s}}$; Infinite Frequency Limit, $\boldsymbol{\varepsilon}_{\infty}$; Relaxation Times, $\boldsymbol{\tau}_{\boldsymbol{j}}$; Dielectric Strength, $\boldsymbol{s}_{\boldsymbol{j}}$; and Relaxation Frequencies, $\boldsymbol{f}_{\boldsymbol{j}}$, for LiTFSI(aq) at Concentration, $\mathbf{c}$; and $298 \mathrm{~K}$. The subscript, $\boldsymbol{j}$, consists of $\boldsymbol{b}, \boldsymbol{s}$, and $\boldsymbol{I P}$, which denote bulk-like water, slow water and ion pair, respectively. (Units: $\mathbf{c}$ in $\mathrm{m} ; \boldsymbol{\kappa}$ in $\Omega^{-1} \mathrm{~m}^{-1} ; \boldsymbol{\tau}_{\boldsymbol{j}}$ in $10^{-12} \mathrm{~s} ; \boldsymbol{f}_{\boldsymbol{j}}$ in $10^{9} \mathrm{~s}^{-1}$.)

\begin{tabular}{|c|c|c|c|c|c|c|c|c|c|c|c|c|}
\hline C & $\boldsymbol{\kappa}$ & $\varepsilon_{s}$ & $s_{b}$ & $\tau_{b}$ & $f_{b}$ & $\boldsymbol{s}_{\boldsymbol{s}}$ & $\tau_{s}$ & $\boldsymbol{f}_{s}$ & $s_{I P}$ & $\tau_{I P}$ & $\boldsymbol{f}_{I P}$ & $\boldsymbol{\varepsilon}_{\infty}$ \\
\hline 0 & 0.02 & 78.61 & 72.87 & 8.35 & 19.06 & & & & & & & \\
\hline 1 & 25.63 & 68.17 & 44.29 & 8.30 & 19.17 & 13.42 & 21.27 & 7.48 & 8.22 & 857.3 & 0.186 & 2.53 \\
\hline 5 & 38.99 & 41.45 & 13.15 & 3.71 & 42.90 & 15.23 & 22.67 & 7.02 & 2.69 & 267.2 & 0.596 & 1.20 \\
\hline 10 & 26.04 & 27.76 & 8.86 & 2.53 & 63.00 & 8.38 & 28.60 & 5.57 & 3.32 & 220.4 & 0.722 & 1.00 \\
\hline 15 & 13.10 & 20.97 & 6.71 & 1.94 & 81.91 & 5.56 & 30.73 & 5.18 & 4.40 & 210.2 & 0.757 & 1.00 \\
\hline 21 & 6.31 & 17.26 & 5.52 & 1.69 & 94.15 & 3.82 & 31.87 & 4.99 & 5.20 & 245.3 & 0.649 & 1.00 \\
\hline
\end{tabular}

Table S6. Fitted parameters of DRS for eutectic electrolytes. Electrical Conductivities, $\boldsymbol{\kappa}$; Static Permittivity, $\boldsymbol{\varepsilon}_{\boldsymbol{s}}$; Infinite Frequency Limit, $\boldsymbol{\varepsilon}_{\infty}$; Relaxation Times, $\boldsymbol{\tau}_{\boldsymbol{j}}$; Dielectric Strength, $\boldsymbol{s}_{\boldsymbol{j}}$; and Relaxation Frequencies, $\boldsymbol{f}_{\boldsymbol{j}}$, for Eutectic(aq) at Concentration, c; and $298 \mathrm{~K}$. The subscript, $\boldsymbol{j}$, consists of of $\boldsymbol{b}, \boldsymbol{s}$, and $\boldsymbol{I P}$, which denote bulklike water, slow water and ion pair, respectively. (Units: $\mathbf{c}$ in $\mathrm{m} ; \boldsymbol{\kappa}$ in $\Omega^{-1} m^{-1} ; \boldsymbol{\tau}_{\boldsymbol{j}}$ in $10^{-12} \mathrm{~s} ; \boldsymbol{f}_{\boldsymbol{j}}$ in $10^{9} \mathrm{~s}^{-1}$.)

\begin{tabular}{|c|c|c|c|c|c|c|c|c|c|c|c|c|}
\hline C & $\boldsymbol{\kappa}$ & $\varepsilon_{s}$ & $\boldsymbol{s}_{\boldsymbol{b}}$ & $\tau_{b}$ & $f_{b}$ & $\boldsymbol{s}_{\boldsymbol{s}}$ & $\tau_{s}$ & $\boldsymbol{f}_{s}$ & $s_{I P}$ & $\tau_{I P}$ & $f_{I P}$ & $\boldsymbol{\varepsilon}_{\infty}$ \\
\hline 3.5 & 4.61 & 40.42 & 11.59 & 4.44 & 35.82 & 21.65 & 19.17 & 8.30 & 3.94 & 510.2 & 0.312 & 3.24 \\
\hline 7 & 3.98 & 27.35 & 7.00 & 3.17 & 50.28 & 12.89 & 25.35 & 6.28 & 4.54 & 501.2 & 0.318 & 2.93 \\
\hline 10 & 2.92 & 20.68 & 5.46 & 2.26 & 70.50 & 9.94 & 27.31 & 5.83 & 2.85 & 251.8 & 0.632 & 2.43 \\
\hline 14 & 1.67 & 17.12 & 3.94 & 1.86 & 85.72 & 7.27 & 28.70 & 5.55 & 3.51 & 201.8 & 0.789 & 2.40 \\
\hline 20 & 0.67 & 17.54 & 3.69 & 1.24 & 128.36 & 5.36 & 31.70 & 5.02 & 6.72 & 378.2 & 0.421 & 1.77 \\
\hline 28 & 0.26 & 14.24 & 3.53 & 0.89 & 178.48 & 3.65 & 28.89 & 5.51 & 5.82 & 364.0 & 0.437 & 1.24 \\
\hline
\end{tabular}




\section{Supplemental references}

1 Tokmakoff, A. Orientational correlation functions and polarization selectivity for nonlinear spectroscopy of isotropic media. I. Third order. J. Chem. Phys. 1996, 105, 112.

2 Lim, J. et al. Nanometric Water Channels in Water-in-Salt Lithium Ion Battery Electrolyte. J. Am. Chem. Soc. 2018, 140, 15661-15667.

3 Jeon, J., Lee, H., Choi, J.-H. \& Cho, M. Modeling and Simulation of Concentrated Aqueous Solutions of LiTFSI for Battery Applications. J. Phys. Chem. C 2020, 124, 11790-11799.

4 (25) Post, S. T. v. d.; Bakker, H. J., The combined effect of cations and anions on the dynamics of water. Phys. Chem. Chem. Phys. 2012, 14 (18), 6280-6288.

5 Loparo, J. J., Roberts, S. T., Nicodemus, R. A. \& Tokmakoff, A. Variation of the transition dipole moment across the $\mathrm{OH}$ stretching band of water. Chem. Phys. 2007, 341, 218-229.

6 Lin, Y. S., Pieniazek, P. A., Yang, M. \& Skinner, J. L. On the calculation of rotational anisotropy decay, as measured by ultrafast polarization-resolved vibrational pumpprobe experiments. J. Chem. Phys. 2010, 132, 174505. 